\title{
Editorial
}

\section{Editorial: Looking Back on a Year as Editor-in-Chief}

A year ago I took on the role of Editor-in-Chief of The Journal of Neuroscience and began my tenure by writing an editorial entitled, "A Commitment to Communication." In that editorial I outlined a number of plans for new initiatives agreed upon by the editorial board, along with my promise to be as transparent as possible about changes and processes at JNeurosci. Since that time, I have written monthly columns (sometimes a little more frequent, sometimes a little less) to update our authors, reviewers, and readers on the changes that have been put in place. At the 1 year point, it is a good time to reflect on the changes that we have made and discuss new plans.

The changes made so far can be grouped into three categories: a new web hosting platform, which allowed for a suite of new features; updated policies to encourage scientific rigor; and expansion of our content by introducing new features.

\section{New web platform}

On October 17, 2016, JNeurosci migrated to a new online site and started publishing articles online within days of acceptance (Fig. 1). Although we always aimed to get peer-reviewed articles in print as quickly as possible, this new initiative has cut the time from acceptance to availability online from $\sim 60 \mathrm{~d}$ to about 2 weeks. This serves the neuroscience community by even more rapidly disseminating peer-reviewed research.

The new platform also allows us to host extended datasets associated with data-rich articles. The logistics of easily accessing this material in a usable way proved to be more technically challenging than originally expected, so it took longer to implement than the Early Release process. We started allowing submission of extended datasets on March 29, 2017 and quickly identified a recently accepted manuscript for which this would be a helpful feature. There was a rapid uptick in submissions with extended data, suggesting that we had been missing the opportunity to host the subset of neuroscience articles that contain results not easily incorporated into the body of a manuscript. We look forward to evolving our extended data policy to make sure we can support the types of data that are being generated across subfields of neuroscience.

Our new platform also offered a more responsive site that is more easily read from any device, and with a more cohesive organization that is easier to navigate. Plus, we can offer the added feature of Alt-metrics, which shows the attention an article has received from a range of sources from social media to blogs and news items.

In addition, we have implemented direct submission from bioRxiv. Authors of manuscripts posted there can submit directly to JNeurosci with one click. We continue to support the rapid dissemination of scientific research and believe that rigorous peer review by JNeurosci can coexist productively with the posting of preprints.

\section{Scientific rigor: reproducibility}

On April 5, 2017, we added new guidelines for statistical reporting to the JNeurosci Instructions for Authors. While our reviewers

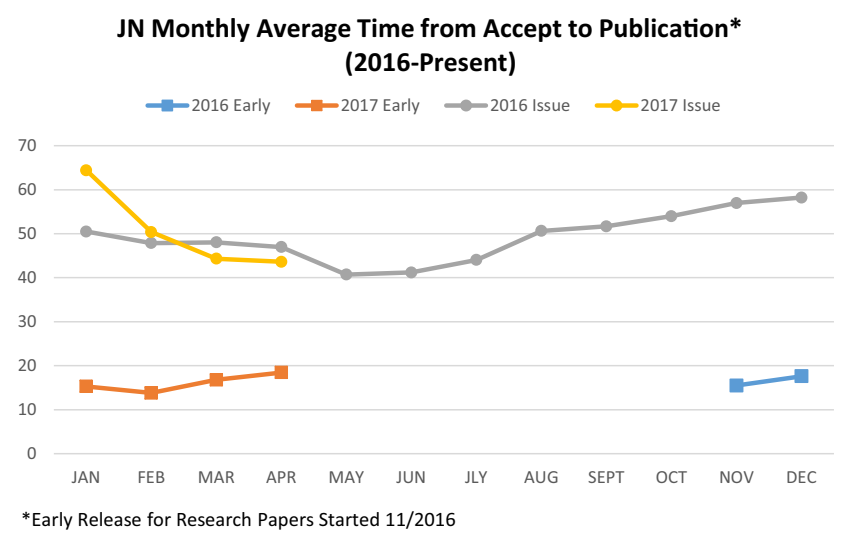

Figure 1. JNeurosci monthly average time from acceptance to publication (2016 to present).

have always commented on aspects of experimental design and statistical analyses when evaluating a manuscript, we wanted to create guidelines that would be relevant across the neuroscience field and support effective evaluation by reviewers that was consistent in its rigorous standards for future replication. To create these guidelines, we convened a group of editors with expertise in different subdisciplines. The updated guidelines derived from these discussions focus on including more details of experimental design and making sure that this information is contained within a single section of the Materials and Methods section, entitled Experimental design and statistical analysis, where it can be found more easily.

Consistent with these efforts to improve rigor and reproducibility, JNeurosci also encourages authors to include RRIDs for critical reagents used in their studies, including antisera, mouse lines, cell lines, etc. The RRID website (https://scicrunch.org/resources) includes links to many reagents used commonly, and links to these identifiers allow other researchers to use common reagents or identify points of divergence in reagents that may explain discrepant findings. Use of RRIDs can also be a way to link key reagents to validation studies. We do not require authors to use RRIDs, but we strongly encourage their inclusion whenever possible.

\section{Feature articles}

In addition to original, peer-reviewed, primary research articles, JNeurosci hosts Feature articles that provide analyses of published research and that put that research into a larger context. We introduced Dual Perspectives to present either alternative points of view on areas of controversy in the neuroscience field or multiple perspectives on scientific issues that can be evaluated at different levels. These articles are paired, and each author is invited to provide a brief commentary on the companion piece after peer review is completed. We would like Dual Perspectives to engage a diverse set of voices in the field and to provide a collegial forum for disagreements in scientific interpretation. 
We also initiated a series of reviews entitled Progressions that present personal views of how an initial finding published in JNeurosci has developed since it first appeared in print. The hope is that these articles highlight not only the scientific journey, but also the personal journey of the neuroscientists who have made seminal discoveries in the field. We have published three Progressions articles so far, and look forward to highlighting additional voices and views.

We will be monitoring the new initiatives put into place over the past year as the articles submitted evolve and take advantage of these innovations. Our goal is to make sure that we are responsive to the needs of our authors, reviewers, and readers and that we take nothing for granted as we look for ways to improve the Journal. As always, please send your thoughts, comments, and ideas to JN_EIC@SFN.org, or you can tweet your ideas to @SfNJournals or@marinap63.

(DMarina Picciotto, EiC, JNeurosci

DOI:10.1523/JNEUROSCI.1386-17.2017 